\title{
Commentary on: Labels, cognomes, and cyclic computation: an ethological perspective
}

\author{
Cedric Boeckx ${ }^{1,2 *}$ and Constantina Theofanopoulou ${ }^{1}$ \\ ${ }^{1}$ Department of General Linguistics, Universitat de Barcelona, Barcelona, Spain, ${ }^{2}$ Catalan Institute for Research and \\ Advanced Studies, Barcelona, Spain
}

Keywords: labeling, cognome, language evolution, computation, animal cognition, cognitive phylogenies

\section{A commentary on}

Labels, cognomes and cyclic computation: an ethological perspective by Murphy, E. (2015). Front. Psychol. 6:715. doi: 10.3389/fpsyg.2015.00715

Murphy (2015) seeks to gain insight into the phylogeny of the human language faculty by adopting a computational perspective. After surveying the ethology literature in an attempt to isolate what is lacking in other species at the computational level, Murphy claims that the process of Labeling, well studied in theoretical linguistics, is what is specific to human language.

We applaud Murphy's effort to address the issue of language phylogeny in such a computationally explicit fashion, but we would like to take this opportunity to highlight a few challenges to building adequate cognitive phylogenies that Murphy does not seem to appreciate enough.

While Murphy's critical remarks toward the "Chomsky hierarchy" as a tool to build biologicallysound cognitive phylogenies (Fitch, 2015) echoes some of our own concerns (Boeckx, 2013; Benítez-Burraco and Boeckx, 2014), we find his appeal to computational principles like Labeling to be equally inadequate. Quite apart from our skepticism coming from narrowly linguistic considerations surrounding Labeling (Boeckx, 2014), our main reason for not siding with Murphy is that issues of cognitive phylogenies must be firmly grounded in comparative studies. But it is well-known that linguistic principles of the sort Murphy trades in resist meaningful comparison across cognitive domains and species.

As Newport (2010: p. 282) has correctly observed, "the generative tradition in language has given us an elegant and detailed articulation of how these principles work themselves out in language; whether the same principles apply in detail to any other domain remains to be seen, since few comparably sophisticated analyses have ever been done of other complex cognitive domains." The generative tradition took some 50 years to arrive at the sophisticated level of computational characterization that Murphy seeks to exploit. Nothing like it exists in other domains of human cognition, let alone in other species. Accordingly, how are we to determine if, say, the "cognome" of baboons contains the Labeling operation when they conceptualize dominance hierarchies? It is not that questions of this type are meaningless, it is just that they are inapplicable in practice, casting doubt on the current feasibility of what Murphy calls computational ethology.

We also find it difficult to believe that operations like Labeling are formulated at the right level of granularity to enable the formulation of linking hypotheses between the cognome and the dynome, connectome, and genome. As argued in Boeckx and Theofanopoulou (2014), notions 
of computation grounded in brain processes, of the sort advocated by Buzsáki (2010) and Buzsáki and Watson (2012), stand a much better chance of providing the right bridging tools between mind and brain. They also can rely on the conservation of brain rhythms across a wide range of species (Buzsáki et al., 2013) to begin to draw meaningful comparisons across species and cognitive domains.

Despite some supporting remarks toward our work, we think that Murphy fails to truly appreciate both the necessity and the primacy of cross-disciplinary, multi-dimensional hypotheses of the sort we have advocated (Boeckx and Theofanopoulou, 2014). In the absence of these, it is quite natural to weaken the Darwinian notion of continuity (as Murphy in fact does with his Weak Continuity Hypothesis), because principles at one level (say, the phenome or cognome) tend to be so specific as to render descent scenarios difficult to articulate. And it is not only this difficulty that worries us, but mainly the fact that phenotypic diversity across species reveals only an apparent gulf (hence, apparent discontinuity) without discernible effects on the cognome. Attempts to detect how such effects percolate downstream succeed to the extent that some traits can indeed be decomposed with a "topdown" approach. But, in our view, it is clear that what is needed instead is a "bottom-up" approach (De Waal and Ferrari, 2010), where sharp differences in the phenome would be conceived as confluences of a hodgepodge of recognizable, reconstructed features. From this standpoint, behavioral-phenotypic experiments across species would only serve as "bootstrapping" bottom-up hypotheses. We are thus convinced that evolution's "tinkering" character-in the spirit of Darwin's notion of "descent"-is to be found in those deeper, more elementary, widely shared traits, as opposed to apparentspecies-specific behaviors. It is in fact this specificity that tends to promote cladistic formulations of cognitive phylogenies (Fitch et al., 2010). But once multi-dimensional hypotheses are formulated, such traditional phylogenetic representations

\section{References}

Benítez-Burraco, A., and Boeckx, C. (2014). Universal grammar and biological variation: an evodevo agenda for comparative biolinguistics. Biol. Theory 9, 122-134. doi: 10.1007/s13752-014-0164-0

Boeckx, C. (2013). Biolinguistics: forays into human cognitive biology. J. Anthropol. Sci. 91, 63-89. doi: 10.4436/jass.91009

Boeckx, C. (2014). Elementary Syntactic Structures: Prospects of a Feature-Free Syntax. Cambridge: Cambridge University Press. doi: 10.1017/CBO9781139524391

Boeckx, C., and Theofanopoulou, C. (2014). A multidimensional interdisciplinary framework for linguistics: the lexicon as a case study. J. Cogn. Sci. 15, 403-420.

Buzsáki, G. (2010). Neural syntax: cell assemblies, synapsembles, and readers. Neuron 68, 362-385 doi: 10.1016/j.neuron.2010. 09.023

Buzsáki, G., Logothetis, N., and Singer, W. (2013). Scaling brain size, keeping timing: evolutionary preservation of brain rhythms. Neuron $80,751-764$. doi: 10.1016/j.neuron.2013.10.002 become untenable (Theofanopoulou, 2015; Theofanopoulou and Boeckx, in preparation).

Consider, for example, the origin of "syllables" in human language, an issue very much related to Labeling. Syllables in language are distinct from "syllables" in the vocal outputs of other species (Samuels, 2011). Accordingly, we could be tempted to posit a human-specific operation-Syllabify-to capture this fact. But work by Ghazanfar and Takahashi (2014) has revealed that a decomposition of syllables in terms of brain rhythms (dynome) favors an evolutionary scenario according to which the mechanism of syllabification is rooted in the mechanism of lipsmacking attested in non-linguistic primates. Computationally speaking, lip-smacking and speech are distinct at the phenome level, but mechanistically (in the dynome) they converge, and provide the basis for elementary cognitive functions (attention, working memory; see Martins and Boeckx, 2014). Murphy's computational ethology approach would be unable to capture it, but the neuroethological approach of Ghazanfar and colleagues does, by resorting to elementary and generic operations framed in brain terms.

In sum, we fully endorse the "divide-and-conquer" approach to cognitive traits that Murphy adopts, but we want to stress the need to recognize that such an approach will only be successful if the "divide" step is combined with a linking step across phenome, cognome, dynome, connectome, and genome. In Aristotle's terms, it is this bridging step that will make us grasp both the Continuity-"in essence" and the Discontinuity-"in appearance."

\section{Acknowledgments}

The present work was made possible through a Marie Curie International Reintegration Grant from the European Union (PIRG-GA-2009-256413), and research funds from the Generalitat de Catalunya (2014-SGR-200), and from the Spanish ministry of economy and competitiveness (FFI201343823-P).

Buzsáki, G., and Watson, B. O. (2012). Brain rhythms and neural syntax: implications for efficient coding of cognitive content and neuropsychiatric disease. Dialogues Clin. Neurosci. 14, 345-367.

De Waal, F., and Ferrari, P. F. (2010). Towards a bottom-up perspective on animal and human cognition. Trends Cogn. Sci. 14, 201-207. doi: 10.1016/j.tics.2010.03.003

Fitch, W. T. (2015). Toward a computational framework for cognitive biology: unifying approaches from cognitive neuroscience and comparative cognition. Phys. Life Rev. 11, 329-364. doi: 10.1016/j.plrev.2014. 04.005

Fitch, W. T., Huber, L., and Bugnyar, T. (2010). Social cognition and the evolution of language: constructing cognitive phylogenies. Neuron 65, 795-814. doi: 10.1016/j.neuron.2010.03.011

Ghazanfar, A. A., and Takahashi, D. Y. (2014). Facial expressions and the evolution of the speech rhythm. J. Cogn. Neurosci. 26, 1196-1207. doi: 10.1162/jocn_a_00575

Martins, P. T., and Boeckx, C. (2014). Attention mechanisms and the mosaic evolution of speech. Front. Psychol. 5:1463. doi: 10.3389/fpsyg.2014. 01463 
Murphy, E. (2015). Labels, cognomes and cyclic computation: an ethological perspective. Front. Psychol. 6:715. doi: 10.3389/fpsyg.2015. 00715

Newport, E. L. (2010). Plus or minus 30 years in the language sciences. Top. Cogn. Sci. 2, 367-373. doi: 10.1111/j.1756-8765.2010. 01102.x

Samuels, B. (2011). Phonological Architecture. Oxford: Oxford University Press. doi: 10.1093/acprof:oso/9780199694358.001.0001

Theofanopoulou, C. (2015). What Cognitive Phylogenies Got Wrong. Tokyo Lectures in Language Evolution. Tokyo: University of Tokyo.
Conflict of Interest Statement: The authors declare that the research was conducted in the absence of any commercial or financial relationships that could be construed as a potential conflict of interest.

Copyright (C) 2015 Boeckx and Theofanopoulou. This is an open-access article distributed under the terms of the Creative Commons Attribution License (CC BY). The use, distribution or reproduction in other forums is permitted, provided the original author(s) or licensor are credited and that the original publication in this journal is cited, in accordance with accepted academic practice. No use, distribution or reproduction is permitted which does not comply with these terms. 